\title{
Functional magnetic resonance imaging in episodic cluster headache
}

\author{
Nicola Morelli · Ilaria Pesaresi - Gianfranco Cafforio · \\ Maria Rosaria Maluccio · Sara Gori · \\ Francesco Di Salle $\cdot$ Luigi Murri
}

Received: 17 September 2008/ Accepted: 26 November 2008/Published online: 16 December 2008

(C) Springer-Verlag 2008

\begin{abstract}
We have investigated the cerebral activation centre in four patients with episodic cluster headache $(\mathrm{CH})$ with functional magnetic resonance imaging (f-MRI). The patients underwent MRI scans for anatomical and functional data acquisition in the asymptomatic state, during a headache attack and after subcutaneous administration of sumatriptan. Anatomical images were acquired by means of 3D-MPRAGE sequences and f-MRI images were obtained by means of echo-planar imaging. Data was analysed using the BrainVoyager QX version 1.7.81 software package. In all patients, the data showed significant hypothalamic activation of the hypothalamus ipsilateral to the pain side, attributable to a headache attack. Overall, we have demonstrated the anatomical location of central nervous system activation by means the first f-MRI study in $\mathrm{CH}$ patients. f-MRI offers a good balance of spatial and temporal resolution, and this method of study appears appropriate for investigating the pathogenetic aspects of primary headaches. Positron emission tomography and f-MRI may be regarded as little or no importance in a
\end{abstract}

N. Morelli $(\bowtie) \cdot$ G. Cafforio · M. R. Maluccio · S. Gori ·

F. Di Salle · L. Murri

University Centre for Adaptive Disorders and Headache

(UCADH), Institute of Neurology, Department

of Neurosciences, University of Pisa, Pisa, Italy

e-mail: n.morelli@inwind.it

N. Morelli

Guglielmo da Saliceto Hospital, Neurology Unit, Piacenza, Italy

I. Pesaresi

Department of Radiology, University of Pisa, Pisa, Italy

F. Di Salle

Department of Cognitive Neurosciences, Faculty of Psychology,

University of Maastricht, Maastricht, The Netherlands clinical context, they do, however, offer great potential for the exploration of headache physiopathology and the effects of pharmacological treatment.

Keywords Functional magnetic resonance imaging . Episodic cluster headache $\cdot$ Hypothalamus

\section{Introduction}

Until recently, primary headaches such as migraine and cluster headache $(\mathrm{CH})$ were described as vasomotor headaches [1]. Initial positron emission tomography (PET) images shed light on the origins of these syndromes, documenting activation in the mesencephalic and pons regions for migraine, and in the grey hypothalamus for $\mathrm{CH}$ and others of trigeminal autonomic cephalalgias (TACs) [2-4].

TACs include $\mathrm{CH}$, paroxysmal headache and Shortlasting Unilateral Neuralgiform headache attacks with Conjunctival injection and Tearing (SUNCT syndrome). It is interesting to note that the common clinical aspects of this primary headaches, where posterior hypothalamic activation has been reported, concern the important cranial autonomic symptoms. The concept of TACs indicates a possible common physiopathological base for these syndromes, not shared with other primary headaches, such as migraine or tension-type headache.

PET and functional magnetic resonance imaging (f-MRI) may be regarded as of little or no importance within a clinical context, but they offer great potential for the exploration of headache physiopathology and the effects of pharmacological treatment [4]. In recent years, fMRI studies have been conducted in TACs, but to date, no studies dealing with $\mathrm{CH}$ are available. In the present study 
we have investigated the cerebral activation centre during a $\mathrm{CH}$ attack in four patients with episodic $\mathrm{CH}$, using f-MRI.

\section{Materials and methods}

We enroled four patients ( $45 \pm 4$ years) diagnosed with episodic $\mathrm{CH}$ in accordance with the International Headache Society (IHS) classification (code 3.1.1) [5].

All patients were in the active phase of the illness, and had an average frequency of two episodes a day. In three patients, one of the two attacks had a very regular recurrence $(2$ p.m.), while in the other patient it happened at night (11 p.m.). The average headache duration, without recourse to treatment, was $110 \pm 12 \mathrm{~min}$. All patients showed autonomic symptoms and there was no family history of headaches.

The patients reported no cardiovascular (hypertension and/or ischaemic cardiopathy), cerebrovascular risk factors, or internal pathological symptoms. All patients reported pain on the left side. A prerequisite for patient enrolment, established on the basis of medical history, was a dramatic response to subcutaneous sumatriptan administration $(6 \mathrm{mg})$. Informed consent was obtained and the study was approved by the local Ethics Committee.

\section{Study design}

The functional study of each individual patient took place in a single continuative session, without interruptions. A priori, the duration of the functional examination was established (the same amount of time for each patient) to be long enough to allow registration of the asymptomatic state, the onset of pain and the pain relief-state (provided by administration of drugs). The patients in the study presented attacks at around the same time each day, and this allowed us to place them in the scanner, 15 min before the onset. When pain occurred they should communicate the onset by pressing buttons on a nonmagnetic button-box.

After $5 \mathrm{~min}$, in order to avoid movements of the patients due to the pain and to induce the state of pain-relief, sumatriptan was subcutaneously injected. The end of attack was communicated using the same push-button controls.

\section{Data acquisition}

Data was acquired on a 1.5 T scanner (Maestro Symphony, Siemens). Functional datasets were obtained by means of a T2* weighted gradient echo-EPI pulse sequence (TR/TE/ FA $3,000 \mathrm{~ms} / 60 \mathrm{~ms} / 30^{\circ}$, TR delay $20 \mathrm{~ms}$, FOV $20 \mathrm{~cm}$, matrix $64 \times 64$, voxel $3.125 \times 3.125 \times 5 \mathrm{~mm}^{3}$, gap $1.25 \mathrm{~mm}$ ). We acquired 20 oblique axial slices, parallel to the anterior-posterior commissure plane, extending from the foramen magnum to the vertex. 400 functional images were acquired. Anatomical datasets were obtained using a T1 MP-RAGE 3D pulse sequence (TR/TI/TE/FA $2,160 \mathrm{~ms} / 1,100 \mathrm{~ms} / 3.93 \mathrm{~ms} / 10^{\circ}$, voxel $1 \times 1 \times 1.4 \mathrm{~mm}^{3}$, gap $0.7 \mathrm{~mm}$ ). 160 oblique sagittal slices were acquired.

Pre-processing of MRI data

Data analysis was carried out using BrainVoyager QX 1.8 software. First, functional data was processed in order to increase the SNR ratio and reduce artefacts. We performed slice scan time correction and 3D motion correction by means of rigid body transformations (the estimated displacement in each direction was less than 3 voxels in each subject) and spatial smoothing with a Gaussian kernel of $4 \mathrm{~mm}$ FWHM (Full-Width-Half-Maximum). Anatomical data was re-sampled to obtain isometric voxels of $1 \times 1 \times 1 \mathrm{~mm}^{3}$ then normalised to the Talairach and Tournaux standard space [6] to allow intersession statistical analysis. Functional and anatomical datasets were co-registered by translation, rotation and zoom operations on the $x, y$ and $z$ axis. Condition specific effects (pain vs. no pain) were estimated using a boxcar approach connected with the hemodynamic response function. Processed functional data obtained from the patients underwent single-subject and multi-subject General Linear Model (GML) analysis. A statistical map was generated as t-contrast with a threshold value of $P<0.001$. Activation was considered significant at a threshold of 0.05 corrected for multiple comparisons.

\section{Results}

During execution of functional MRI (three afternoon sessions and one night session) all the patients suffered from $\mathrm{CH}$ attacks, and the administration of sumatriptan brought pain relief after $4 \pm 1 \mathrm{~min}$ (average values and standard deviation). In all attacks (single-subject GML study), comparing the pain state with the pain-free state, we detected significant cerebral activation $(P<0.05$, corrected for multiple comparisons) in the ipsilateral hypothalamic grey matter (Fig. 1). The coordinates and the $Z$-score of the activation zones are listed in Table 1 . Figure 2 shows the temporal course of the BOLD signals in the hypothalamus ipsilaterally to the pain-side in patient 2 . Additionally, with a less conservative threshold of $P<0.001$ uncorrected, trends of activation were observed in the pre-frontal cortex, anterior cingulate cortex, contralateral thalamus, ipsilateral basal ganglia and bilaterally in the insula and the cerebellar hemispheres. However, these areas were not subject to more detailed analysis because the study was not designed to investigate the activation of the pain matrix (stereotactic co-ordinates were not shown). 
Fig. 1 Multi-subject GML analysis shows cerebral activation area in the posterior grey hypothalamus, ipsilateral to the pain side $(P<0.001$ uncorrected for descriptive purposes), superimposed on a T1-weighted anatomical reference MR image in axial (a) and coronal (b) planes
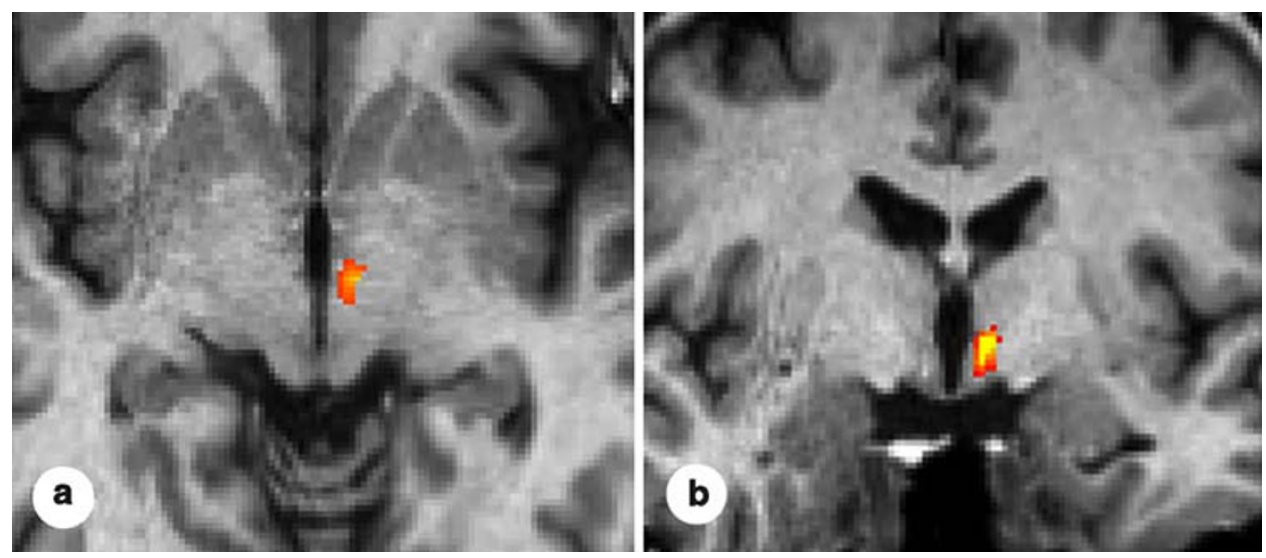

Table 1 BOLD-signal increases during spontaneous $\mathrm{CH}$ attack compared with the pain-free state $(P<0.05$, corrected for multiple comparisons)

\begin{tabular}{lrrrl}
\hline & \multicolumn{2}{l}{ Talairach coordinates } & \multicolumn{2}{l}{$Z$ Z-score } \\
\cline { 2 - 3 } & \multicolumn{1}{l}{$x$} & \multicolumn{1}{c}{$y$} & \multicolumn{1}{c}{$z$} & \\
\hline Patient 1 & -6 & -10 & 1 & 4.89 \\
Patient 2 & -3 & -8 & -1 & 4.83 \\
Patient 3 & -5 & -6 & 4 & 4.91 \\
Patient 4 & -4 & -7 & 2 & 4.32 \\
Multi-subject study & -5 & -8 & -1 & 6.93 \\
\hline
\end{tabular}

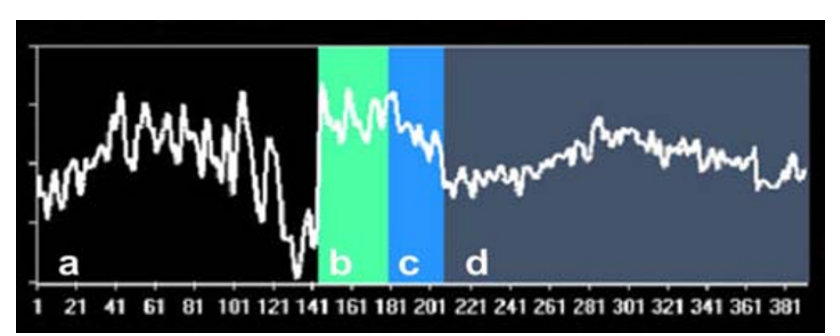

Fig. 2 Temporal course of the BOLD signals in the hypothalamus ipsilaterally to the pain-side in patient 2. Asymptomatic state (a). Onset of the headache attack and state of pain (b). Administration of sumatriptan (c). Pain relief-state (d). On the horizontal line the number of the 400 GE-EPI acquisitions, is indicated

\section{Discussion}

This study documents the activation of cerebral areas normally attributed to the analysis of painful stimuli (prefrontal cortex, basal ganglia, thalamus, cingulate cortex, insula and cerebellum) as reported in a comprehensive review [7] and of the hypothalamic region ipsilaterally to the pain-side as documented in previous functional studies carried out using PET methods [3] in CH. To date, f-MRI has only been used in the study of patients affected by other TACs. [8] Previously, in short-lasting unilateral neuralgiform headache attacks with conjunctival injection and tearing, f-MRI studies have shown both hypothalamic activation ipsilateral to the pain side [9] and bilateral activation of the hypothalamus [10]. In one case of atypical TAC [11], activation of the hypothalamus, ipsilateral to the pain side, has been reported using f-MRI .

Overall, we have demonstrated the anatomical location of central nervous system activation with the first f-MRI study during a $\mathrm{CH}$ attack. The diencephalon-hypothalamic region seems to be an activation area specific to $\mathrm{CH}$, and for this reason, the possible role of attack generator centres has been suggested. As suggested by clinical, biohumoral and electrophysiological data, the hypothalamus plays a role in determining biological rhythms [12, 13]. There is great need to investigate $\mathrm{CH}$ and other related idiopathic headache syndromes using f-MRI which offers a good balance of spatial and temporal resolution. The f-MRI presents major advantages in comparison with the PET: it does not use radioisotope administrative tracers (intravenously), but endogenous tracers (DeoxyHaemoglobin). Furthermore f-MRI allows to effect anatomical and functional acquisitions in the same recording session.

This study method seems appropriate for investigation of pathogenetic aspects in the primary headaches, particularly for analysis of functional data immediately prior to attack onset.

\section{Conflict of interest None.}

\section{References}

1. Wolff HG (ed) (1963) Headache and other headache pain, 2nd edn. Oxford University Press, New York

2. Weiller C, May A, Limmroth V (1995) Brain stem activation in spontaneous human migraine attacks. Nat Med 1:658-660

3. May A, Bahra A, Buchel C, Frackowiak RSJ, Goadsby PJ (1998) Hypotalamic activation in cluster headache attacks. Lancet 352:275-278

4. Sánchez del Rio M, Alvarez Linera J (2004) Functional neuroimaging of headaches. Lancet Neurol 3(11):645-651 
5. Headache Classification Committee of the International Headache Society (2004) Classification and diagnostic criteria foe headache disorders, cranial neuralgias and facial pain, 2nd edn. Cephalalgia 24:1-160

6. Talaraich J, Tournaux P (1988) Coplanar stereotaxic atlas of the human brain. Thieme, New York

7. Bingel U, Quante M, Knab R et al (2002) Subcortical structures involved in pain processing: evidence from single trial fMRI. Pain 99:313-321

8. Matharu M, May A (2008) Functional and structural neuroimaging in trigeminal autonomic cephalalgias. Curr Pain Headache Rep 12(2):132-137

9. May A, Bahra A, Büchel C, Turner R, Goadsby PJ (1999) Functional magnetic resonance imaging in spontaneous attacks of SUNCT: short-lasting neuralgiform headache with conjunctival injection and tearing. Ann Neurol 46(5):791-794
10. Sprenger T, Valet M, Platzer S, Pfaffenrath V, Steude U, Tolle TR (2005) SUNCT: bilateral hypothalamic activation during headache attacks and resolving of symptoms after trigeminal decompression. Pain 113(3):422-426

11. Sprenger T, Valet M, Hammes M, Erhard P, Berthele A, Conrad B, Tolle TR (2004) Hypothalamic activation in trigeminal autonomic cephalgia: functional imaging of an atypical case. Cephalalgia 24(9):753-757

12. Hastings MH, Herzog ED (2004) Clock genes, oscillators, and cellular networks in the suprachiasmatic nuclei. J Biol Rhythms 19(5):400-413

13. Moore RY (1997) Circadian rhythms: basic neurobiology and clinical applications. Annu Rev Med 48:253-266 